\title{
The Environments of the Massive Star Clusters in the Carina Nebula
}

\author{
Kate Brooks \\ European Southern Observatory, Casilla 19001, Santiago 19, Chile \\ Jill Rathborne, Michael Burton \\ School of Physics, University of New South Wales, Sydney 2052, NSW, \\ Australia
}

\begin{abstract}
We have imaged over an extensive field the 3.29- $\mu \mathrm{m}$ emission from the Carina Nebula. This emission arises from polycyclic aromatic hydrocarbons which are excellent tracers of photodissociation regions. The results emphasis the two very different environments of the massive star clusters of the nebula, Trumpler 14 and 16.
\end{abstract}

\section{Introduction}

The feedback processes from a cluster of young, massive stars have a profound impact on their natal giant molecular cloud (GMC), particularly on its future star-forming capacity. The Carina Nebula, at a distance of $2.2 \mathrm{kpc}$, is one of the most extreme and complex cases to study in detail such feedback processes. It contains two of the most massive star clusters in our galaxy, Trumpler 14 and Trumpler 16 ( $\operatorname{Tr} 14$ and $\operatorname{Tr} 16$ ), which contain a combined total of 33 O-type stars (e.g. Tovmassian 1995). Tr 16 also contains one of the most massive stars known, Eta Carinae. The associated GMC consists of two components known as the northern and southern clouds (e.g. Brooks, Whiteoak, \& Storey 1998). Tr 14 is partially embedded in the northern cloud whereas $\operatorname{Tr} 16$ is situated in between the two clouds and associated with the bright Keyhole Nebula.

We have imaged over a 30 arcmin field the Carina Nebula through a narrowband filter centred at $3.29 \mu \mathrm{m}$ using the SPIREX/Abu telescope at the South Pole. This emission arises from polycyclic aromatic hydrocarbons (PAHs) which are excellent tracers of photodissociation regions (PDRs) (e.g. Hollenbach \& Tielens 1999). The wide field of view allows us to study the resulting interaction across the star forming complex, while still retaining sufficient spatial resolution to examine the structure across the PDR interfaces.

\section{3.29- $\mu \mathrm{m}$ PAH emission}

Figure 1 shows the 3.29-um PAH emission observed across the Carina Nebula. The wide-spread emission can be grouped into three main regions: northern cloud region, Keyhole Nebula and southern cloud region. 


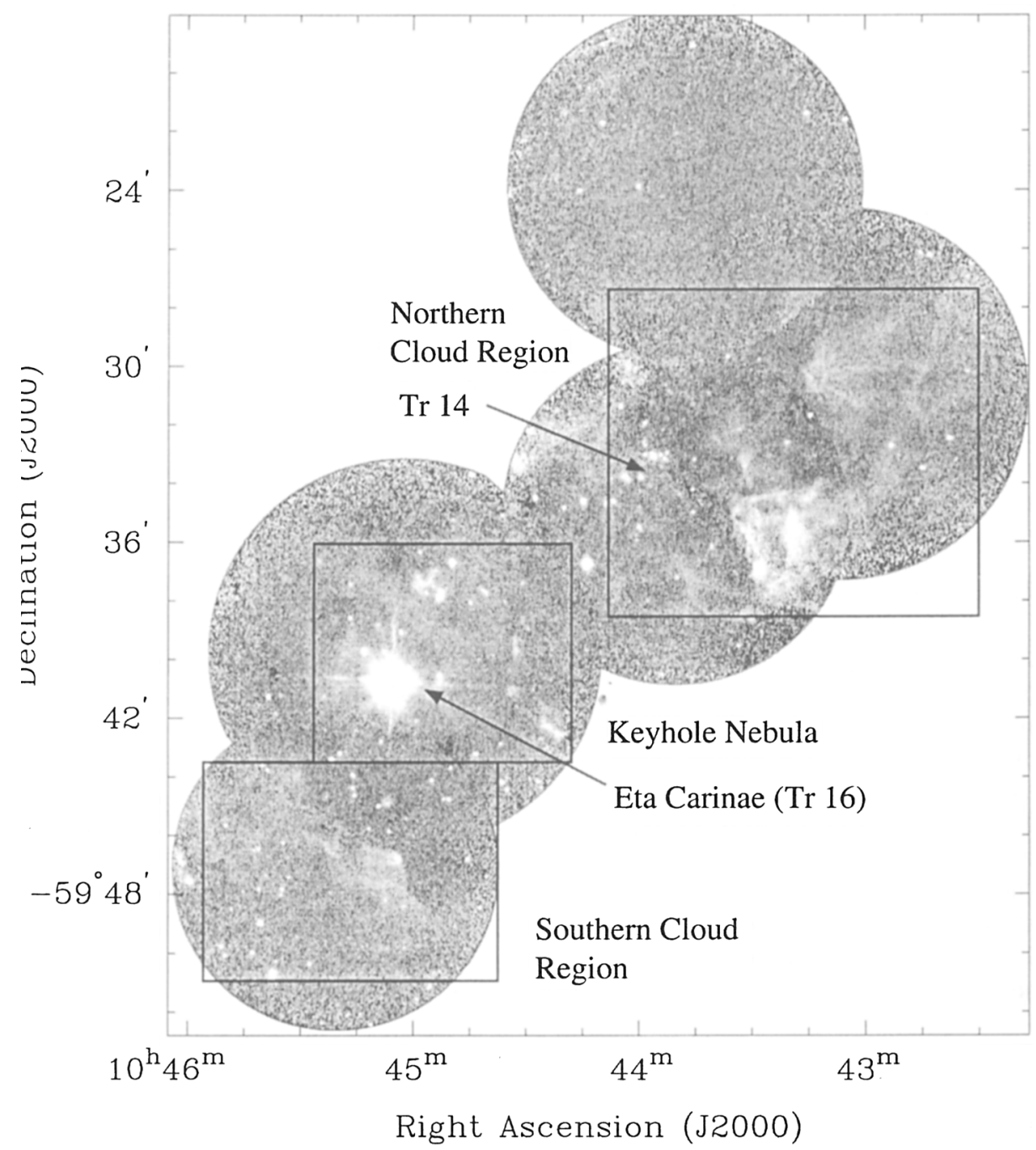

Figure 1. Narrow-band 3.29- $\mu \mathrm{m}$ emission towards the Carina Nebula. Note that the image includes a continuum emission component. Based on the results of L-band imaging at $3.5 \mu \mathrm{m}$ the continuum emission is largely confined to stellar emission and does not contribute significantly to the narrow-band emission. This work is reported in more detail in Rathborne et al. 2002 (in preparation). 
For the northern region, the brightest PAH emission concentration is situated west of $\operatorname{Tr} 14$ and is coincident with a dense part of the northern molecular cloud. This emission is almost a factor of ten times higher in brightness than the emission observed in the other regions, reaching a peak intensity of $40 \times 10^{-14} \mathrm{erg} \mathrm{s}^{-1} \mathrm{~cm}^{-2} \operatorname{arcsec}^{-2}$. The prominently curved eastern edge of this emission is adjacent to a bright ionization arc belonging to the HII region Car I (Brooks, Storey, \& Whiteoak 2001). Car I is ionized by Tr 14 and is thought to be expanding into the molecular cloud and carving out an ionized cavity. Such widespread PDR emission is a direct consequence of the penetrating radiation field from $\operatorname{Tr} 14$ and of the clumpy nature of the molecular gas.

In contrast, the observed PAH emission in the vicinity of $\operatorname{Tr} 16$ and the Keyhole Nebula is confined to several distinct clumps. These clumps have previously been studied at millimeter and infrared wavelengths and found to be molecular gas clumps of around $10 \mathrm{M}_{\odot}$ and with PDRs on their surface (Brooks et al. 2000; Cox \& Bronfman 1995). They appear to have been swept up from molecular gas by the stellar winds originating from $\operatorname{Tr} 16$ and are now being over-run by the intense ionization field which is photo-evaporating the clumps.

The diffuse PAH emission observed in the southern region traces the southern molecular cloud. In particular, the prominent emission rim traces the northwestern edge of the cloud. The brightest PAH emission peak is coincident with the source IRAS10430-5931 and a bright-rimmed molecular globule. Several sources displaying anomalous near-infrared colours exist at the edge of this globule and are thought to be the first evidence of ongoing star formation in the Carina Nebula (Megeath et al. 1996).

\section{Summary}

The bright PAH emission detected in the northern part of the Carina Nebula shows that the radiation field from $\operatorname{Tr} 14$ has penetrated the nearby molecular cloud and has created wide-spread PDRs. In contrast, the emission surrounding Tr 16 is confined to several small molecular clumps which are likely to be remnants of the molecular cloud which formed $\operatorname{Tr} 16$. They may represent a future state for the northern molecular cloud. Such a scenario is consistent with $\operatorname{Tr} 14$ being younger $\left(1 \times 10^{6} \mathrm{yr}\right)$ than $\operatorname{Tr} 16\left(3 \times 10^{6} \mathrm{yr}\right)$.

\section{References}

Brooks, K. J., Storey, J. W. V., \& Whiteoak, J. B. 2001, MNRAS, 327, 46

Brooks, K. J., Burton, M. G., Rathborne, J. M., Ashley, M. C. B., \& Storey, J. W. V. 2000, MNRAS, 319, 95

Brooks, K. J., Whiteoak, J. B., \& Storey, J. W. V. 1998, PASA, 15(2), 202

Tovmassian, H. M. 1995, RevMexAA (Serie de Conferencias), 2, 83

Hollenbach, D., \& Tielens, A. G. G. M. 1999, Rev Mod Phys, 71, 173

Cox, P., \& Bronfman, L. 1995, A\&A, 299, 583

Megeath, S. T., Cox, P., Bronfman, L., \& Roelfsema, P. R. 1996, A\&A, 305, 296 